\title{
Allocation of Local and Global Search Capabilities of Particle in Canonical PSO
}

\author{
Junqi Zhang Kun Liu Ying Tan* Xingui He \\ Key Laboratory of Machine Perception (Peking University), Ministry of Education, and Department of \\ Machine Intelligence, School of Electronics Engineering and Computer Science, Beijing, China \\ ytan@pku.edu.cn
}

\begin{abstract}
This paper analyzes theoretically the exact sampling distribution of the particle swarm optimization (PSO) without any assumption imposed by all current analyses. The distribution of particles in the PSO in one-step transition is analyzed in details. Especially, local and global search capabilities of particles in the PSO are defined implicitly, and are allocated adaptively to show how the PSO works by several experiments. In essence, the PSO works by just allocating each particle in the swarm to finish two jobs in probability, locally searching the range around the current best positions and globally searching whole solution space. According to our definitions and analyses, the exact probabilities of the two jobs can be measured by theoretic derivations and experiments whilst how the PSO allocate the particles' search capabilities can be recognized clearly. So we can look into the PSO in depth.
\end{abstract}

Categories and Subject Descriptors: I.2.8 [Artificial Intelligence]: Problem Solving, Control Methods, and Search

General Terms: Theory, Performance.

Keywords: Particle Swarm Optimization (PSO), Sampling Distribution, Global Search, Local Search, Allocation, Guide.

\section{INTRODUCTION}

The particle swarm optimization (PSO) is a stochastic global optimization technique inspired by social behavior of bird flocking or fish schooling $[1,2]$. The sampling distribution of the PSO is analyzed under the assumption of stagnation in [3,4]. Even though all current analyses rely on simplifications or assumptions that the particles are deterministic or stagnated, this paper analyzes theoretically the exact sampling distribution of the PSO without any simplification, which can help us understand how the PSO works very much clearly.

In conventional PSO, the update formula for each particle's velocity and position in conventional standard PSO is written as

$$
\begin{array}{r}
V_{i d}(t+1)=w V_{i d}(t)+c_{1} r_{1}\left(P_{i B d}(t)-X_{i d}(t)\right) \\
+c_{2} r_{2}\left(P_{g B d}(t)-X_{i d}(t)\right), \\
X_{i d}(t+1)=X_{i d}(t)+V_{i d}(t+1)
\end{array}
$$

\section{SAMPLING OF PSO}

As we know, in each iteration, PSO gives every particle two guides to evolve, which are $P_{i B d}(t)$ and $P_{g B d}(t)$. So, attention

${ }^{*}$ Corresponding author.

Copyright is held by the author/owner(s).

GECCO'08, July 12-16, 2008, Atlanta, Georgia, USA.

ACM 978-1-60558-130-9/08/07. should be paid on these two guides to find how exactly they change the distribution of one particle in the search space to find the actual solution with higher probability. We denote $\varphi_{1}=c_{1} r_{1}, \varphi_{2}=$ $c_{2} r_{2}, d_{1}=P_{p B}(t)-X(t), d_{2}=P_{g B}(t)-X(t), a=\min \left\{c_{1} d_{1}\right.$, $\left.c_{2} d_{2}\right\}, b=\max \left\{c_{1} d_{1}, c_{2} d_{2}\right\}, m=\operatorname{argmin}_{i}\left\{c_{i} d_{i}\right\}, i=1,2$, $n=\operatorname{argmax}_{i}\left\{c_{i} d_{i}\right\}, i=1,2, A\left(t=\varphi_{m} d_{m}, B(t)=\varphi_{n} d_{n}\right.$, $Z(t)=A(t)+B(t)$.

The distribution function of $\mathrm{Z}$ is

$f_{Z}(z)=\int_{-\infty}^{+\infty} f_{Z, A}(z, x) d x=\int_{-\infty}^{+\infty} f_{A}(x) * f_{B}(z-x) d x$

\subsection{Case $a \geq 0$}

$$
f_{Z}(z)= \begin{cases}0 & \text { if } z>(a+b) \quad \text { or } \quad z \leq 0, \\ \frac{z}{a b} & \text { if } 0<z \leq a, \\ \frac{1}{b} & \text { if } a<z \leq b, \\ \frac{a+b-z}{a b} & \text { if } b<z \leq(a+b) .\end{cases}
$$

2.2 Case $a<0$ and $b>0$

$$
f_{Z}(z)= \begin{cases}0 & \text { if } z>b \text { or } z<a \\ \frac{z-a}{|a| b} & \text { if } a \leq z \leq \min \{0, a+b\} \\ \frac{1}{|a|} & \text { if }(a+b)<z \leq 0 \text { and } \quad(a+b)<0 \\ \frac{1}{b} & \text { if } 0<z \leq(a+b) \text { and } \quad(a+b) \geq 0 \\ \frac{b-z}{|a| b} & \text { if } \max \{a+b, 0\}<z \leq b .\end{cases}
$$

When $(a+b) \geq 0$, Eq. (5) can be written as

$$
f_{Z}(z)= \begin{cases}0 & \text { if } z>b \text { or } z<a \\ \frac{z-a}{|a| b} & \text { if } a \leq z \leq 0 \\ \frac{1}{b} & \text { if } 0<z \leq(a+b), \\ \frac{b-z}{|a| b} & \text { if }(a+b)<z \leq b .\end{cases}
$$

When $(a+b)<0$, Eq. (5) can be written as

$$
f_{Z}(z)= \begin{cases}0 & \text { if } z>b \text { or } z<a \\ \frac{z-a}{|a| b} & \text { if } a \leq z \leq(a+b) \\ \frac{1}{|a|} & \text { if }(a+b)<z \leq 0 \\ \frac{b-z}{|a| b} & \text { if } 0<z \leq b\end{cases}
$$

\subsection{Case $b \leq 0$}

$$
f_{Z}(z)= \begin{cases}0 & \text { if } z<(a+b) \quad \text { or } \quad z \geq 0 \\ \frac{z-a-b}{|a b|} & \text { if }(a+b)<z \leq a, \\ \frac{1}{|a|} & \text { if } a<z \leq b, \\ \frac{|z|}{a b} & \text { if } b<z \leq 0 .\end{cases}
$$




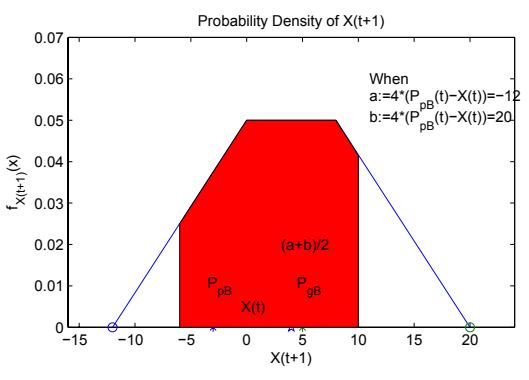

Figure 1: The allocation of $P($ local $)$ (The deep color area) and $P($ global $)$ (The rest area of the trapezia) when there is no inertia velocity part.

\subsection{How does PSO work}

$$
X(t+1)=X(1)+\sum_{i=1}^{t} w^{i} V(1)+\sum_{j=1}^{t-1} \sum_{i=0}^{j} w^{i} Z(t-i)
$$

\section{ALLOCATION OF GLOBAL EXPLORATION AND LOCAL EXPLOITATION}

Definition 1(Local Search Range): The sampling range of a particle $\mathrm{X}$ for local search is defined as:

$$
\begin{array}{r}
R(\text { local })=\left[\left(P_{p B}-\left|d_{1}\right|\right),\left(P_{p B}+\left|d_{1}\right|\right)\right] \\
\bigcup\left[\left(P_{g B}-\left|d_{2}\right|\right),\left(P_{g B}+\left|d_{2}\right|\right)\right] .
\end{array}
$$

Definition 2(Global Search Range): The sampling range of a particle $\mathrm{X}$ for global search is defined as:

$$
R(\text { global })=R-R(\text { local }) .
$$

where $R$ denotes a set of all real numbers.

Definition 3(Local Search Probability): The probability of a particle $X$ landing in $\mathrm{R}$ (local) is defined as:

$$
P(\text { local })=\int_{R(\text { local })} f_{X}(x) d x .
$$

Definition 4(Global Search Probability): The probability of a particle $X$ landing in $\mathrm{R}$ (global) is defined as:

$$
P(\text { global })=\int_{R(\text { global })} f_{X}(x) d x .
$$

As can be seen by Eqs. (12) and (13), there exists

$$
P(\text { global })+P(\text { local })=1 .
$$

For the sake of precision, the $P($ local $)$ and $P($ global $)$ of every generation in every dimension of each particle can be integrated precisely according to Eqs.(10), (11) and (13) and shown in Fig. 1.

In the similar manner, we compute the global allocation probability of the PSO without and with the inertial velocity part during the evolution shown in Fig 2. The range of the sampling distribution for $\mathrm{Z}$ will be shifted according to the value of $w * V(t)$ and the global search is increased.

In order to look into the behavior of the canonical PSO in the stagnation, we set the best fitness value of the swarm as a fixed and same constant in every generation. $P($ global $)$ is illustrated in Fig. 3. As can be seen, $P($ global $)$ decreases with the evolving

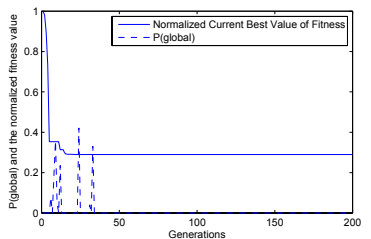

(a) without inertia velocity part

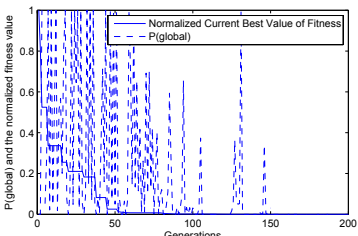

(b) with inertia velocity part
Figure 2: The $P($ global $)$ of PSO without and with inertia velocity part using three particles with three dimensions on Ackley Function, where $c_{1}=c_{2}=2$. The Best value of the fitness found is 16.7 and 0.0001 respectively, and stagnation begins after the $46 t h$ and 161 st generation respectively.

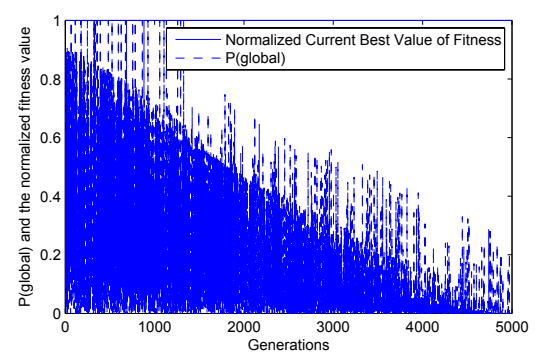

Figure 3: The $P($ global $)$ of PSO with the inertia velocity part on Ackley function, where $c_{1}=c_{2}=2$. $w=\mathbf{1}$, total number of generations is $\mathbf{5 0 0 0}$.

process. Therefore, as the stagnation happened, the canonical PSO trends to pay more attentions on local search.

\section{Acknowledgment}

This work is partially supported by the National High Technology Research and Development Program of China (863 Program), with grant No.2007AA01Z453, and partially supported by the National Natural Science Foundation of China under grant No.60673020. This work is also financially supported by the Research Fund for the Doctoral Program of Higher Education (RFDP) in China and and China Postdoctoral Science Foundation funded project grants No.20070420257.

\section{REFERENCES}

[1] J. Kennedy and R. Eberhart, Particle Swarm Optimization, Proceedings of the IEEE International Conference on Neural Networks, 1995, pp. 1942-1948.

[2] R.C.Eberhart and J.Kennedy, A new optimizer using particle swarm theory, Proceedings of the 6th Int. Symp. Mcro Machine Human Science, 1995, 39-43.

[3] J. Kennedy and David Broomhead, Exact analysis of the sampling distribution for canonical particle swarm optimiser and its convergence during stagnation, Proceedings of the IEEE International Conference on Genetic And Evolutionary Computation Conference, 2007, 134-141.

[4] J. Kennedy, On the moments of the sampling distribution of particle swarm optimisers, Proceedings of the IEEE International Conference on Genetic And Evolutionary Computation Conference, 2007, 2907-2914. 